\section{Un instrumento} de factores asociables, en el marco de la evaluación de competencias básicas en el D.C.

An Instrument of Associable Factors in the Context of Basic Competences Assessment in the Distrito Capital

Um instrumento de fatores associáveis, no marco da avaliação de competências básicas no Distrito Capital

\section{Guillermo Bustamante Zamudio* Luis Guillermo Diaz Monroy**}

\footnotetext{
* Doctor en Educación por la Universidad Pedagógica Nacional; Magíster en lingüística y español por la Universidad del Valle. Profesor de la Universidad Pedagógica Nacional. Correo: guibuza@gmail.com

** Candidato a doctor en estadística por la Universidad Nacional de Colombia; Magíster en estadística por la misma universidad. Profesor de la Universidad Nacional de Colombia. Correo: lgdiazm@unal. edu.co
}

\section{Resumen}

Inscrita en lógicas de eficiencia y diseño técnico de la educación, la evaluación parece modular efectos importantes que van más allá de un simple horizonte cognitivo o de un propósito ético. Examinar factores asociados al logro de los estudiantes permite detectar novedades, reiteraciones, causalidades y argumentos desde los cuales se pretende objetivar las decisiones políticas en educación. El artículo hace un análisis cualitativo al instrumento de factores asociados aplicado en Bogotá y delata críticamente sus limitaciones y ambigüedades.

\section{Palabras clave}

Evaluación, competencias, factores asociados, desempeño, aprestamiento.

\section{Abstract}

Inscribed in the logic of efficiency and technical design of education, assessment seems to modulate important effects which go beyond a simple cognitive horizon or an ethical purpose. Examining factors associated to the students' achievement allows detecting news, reiterations, causalities and arguments from which it is intended to make objective the political decisions on education. The article makes a qualitative analysis to the associated factors instrument applied in Bogotá and it critically reveals its limitations and ambiguities.

\section{Key words}

Assessment, competences, associated factors, performance, readiness.

\section{Resumo}

Inscrita nas lógicas de eficiência e desenho técnico da educação, a avaliação ao que parece modula efeitos importantes que vão além de um simples horizonte cognitivo ou de um propósito ético. Examinar fatores associados ao rendimento dos estudantes permite detectar novidades, reiterações, causalidades e argumentos desde os quais se pretende objetivar as decisões políticas na educação. 0 artigo realiza uma análise qualitativa do instrumento de fatores associados aplicado em Bogotá e expõe criticamente suas limitações e ambiguidades.

\section{Palavras chave}

Avaliação, competências, fatores associados, desempenho, aprestamento.

Fecha de recepción: Octubre 31 de 2014 Fecha de aprobación: Noviembre 14 de 2014

.................................................... 


\section{Presentación}

$\mathrm{E}$ n Colombia, antes de la década del noventa del siglo pasado, los principales referentes de la política educativa en apariencia eran algunas "intuiciones" sobre aquello que supuestamente le convenía a la educación (no sin los correspondientes ajustes a asuntos fiscales). Pero, a partir de 1991 -y por exigencia de las agencias multilaterales-, la política educativa se diseña "técnicamente", con el propósito explícito de maximizar la eficiencia de la inversión, es decir: con apoyo en estudios estadísticos que cruzan desempeños de los estudiantes (para lo cual se hacen evaluaciones masivas) y factores asociables (para lo cual se aplica una batería específica de preguntas a los mismos estudiantes que desarrollan las pruebas de desempeño).

En 1998, la Secretaría de Educación Distrital de Bogotá (SED) se utilizó para ensayar el paso de aplicaciones muestrales de las evaluaciones masivas (que se realizaban desde 1991 a escala nacional), a aplicaciones censales. Se hizo entonces una evaluación de desempeño en lenguaje y matemáticas (diseñada por la Universidad Nacional de Colombia) y un estudio sobre factores asociados al logro de los estudiantes (diseñado por la Misión Social del Departamento Nacional de Planeación, DNP, cuyo informe final se tituló: Evaluación de la calidad de la educación primaria en Santafé de Bogotá 1998 - Factores asociados al logro).

Los autores del presente artículo veníamos de realizar con el IDEP, entre 1999 y 2000, la investigación "Análisis de algunos factores asociables al desempeño en el área de lenguaje: el D.C. frente al resultado nacional", que utilizaba datos de una prueba muestral hecha por el Ministerio de Educación Nacional, como requisito para poner término al "Plan de universalización de la educación básica primaria". Propusimos entonces a la Universidad Nacional la investigación titulada: "Factores asociados en la evaluación del D.C.", ahora con los datos de la evaluación censal. Nuestra propuesta fue aprobada para el período 2000-2001. De ella hace parte el este artículo, en el que hacemos un análisis cualitativo de algunos aspectos del formulario de "factores asociados" aplicado por el DNP.

\section{El formulario}

El formulario está constituido de un conjunto de frases como las siguientes (tomamos algunas de entre las 37 preguntas del instrumento aplicado a los alumnos):
- ¿De qué sexo eres?

- ¿Cuál o cuáles cursos has repetido?

- ¿En qué vienes al colegio todos los días?

- ¿Te ponen tareas de lenguaje para hacer en la casa?

- Las personas de tu familia, ¿en cuántos cuartos duermen?

- Tu papá, tu mamá, o la persona que te cuida, ¿sabe o no leer y escribir?

- La mayor parte del piso de tu casa (o apartamento) es de: [se dan las opciones]

Estos enunciados son asumidos por los evaluados en forma diversa (Velázquez, 1995). En tanto la comunicación no está conformada simplemente por personas "reales", sino por roles y por asignación imaginaria de características a esos roles, la actitud podría ser la de preguntarse qué quiere el encuestador y, en consecuencia, responder con base en esa expectativa; por ejemplo, responder como si el encuestador tuviera necesidad de esa información y sus intenciones estuvieran claras a lo largo del cuestionario. Ante preguntas en las que es evidente el estereotipo y toda distancia con respecto a él corre el riesgo de ser sancionada negativamente, ¿quién respondería otra cosa que no sea lo conveniente?

Esto produce una información heterogénea que no va a ser tenida en cuenta, pues, para efectos de la "investigación" estadística, las posiciones frente al evaluador se consideran homogéneas; de lo contrario, la información obtenida con el instrumento no sería confiable (Simiand, 1994). Pero, ¿y si esas posiciones son determinantes? Es el caso del "encuadramiento" que han encontrado los mismos que se dedican a la psicometría: ante las mismas preguntas, pero en orden diverso, las respuestas de los encuestados pueden variar notablemente ${ }^{1}$.

Entonces, ¿acaso lo que el instrumento "mide" es la capacidad del encuestado para enfrentar la pragmática de la prueba?; recordemos lo que advierte Stubbs (1984): la posición en la que dos niños de

1 Oliverio (1998) informa sobre el experimento de unos psicólogos norteamericanos; ante la pregunta: "Un país comunista como la URSS, ¿debería permitir a los periodistas norteamericanos difundir en Norteamérica aquellas noticias que consideren oportunas sin censurarlas?", el $82 \%$ de los encuestados responde que sí; $y$, al abordar la siguiente pregunta: “¿Deberían permitir los EE. UU. a los periodistas rusos difundir las noticias tal y como las ven y juzgan bajo su óptica particular", el $75 \%$ responde que sí. Pero cuando se invierte el orden de las preguntas, los porcentajes bajan a $65 \%$ y $55 \%$, respectivamente. 
estrato social distinto van a una encuesta en los Estados Unidos no depende de su inteligencia o de su competencia, sino de su posición frente al evaluador y al contexto.

Por todo esto, frente a los instrumentos de factores asociados caben las preguntas de Velázquez (1995, p. 2), de un lado, acerca del tipo de ejercicio de visibilidad sobre la educación colombiana que se hace con el instrumento, sobre todo cuando viene de una instancia oficial (en nuestro caso, la Alcaldía, la SED, organismos que constituyen una fuerza en la educación distrital). Y, de otro lado, sobre si el análisis estadístico produce nuevas proposiciones sobre la educación colombiana o si solo justifica y apoya algunas ya existentes.

\section{El formulario como enunciado}

El instrumento va encabezado así: "Alcaldía Mayor de Santafé de Bogotá, D.C. Secretaría de Educación. Evaluación de Competencias y Saberes básicos en Lenguaje y Matemática". Ante esto, quien lo diligencia puede pensar que la Alcaldía y la SED impulsan las competencias y los saberes básicos, que les interesa especialmente la matemática y el lenguaje. De modo que todo lo que se diga en el formulario apunta a eso y, además: a. de una manera fácil ("Las preguntas son sencillas y podrás contestarlas fácilmente", dice el instrumento); b. sin lugar a error ("En esta parte [factores asociables] no hay respuestas buenas ni malas", informa el instrumento); y c. en la que se levanta una prohibición que regía para la prueba de competencias que se acaba de contestar ${ }^{2}$ ("si no entiendes alguna pregunta, pide ayuda a la persona que te entregó el cuestionario", dice el instrumento).

O sea, este tipo de instrumentos puede ser leído como si fuera un solo enunciado: "Las competencias (o la calidad, o lo que sea) dependen de..." y sigue un listado de factores que determinan los buenos o los malos desempeños de los estudiantes. Por supuesto, formulados en como interrogantes, pero no necesariamente una pregunta encarna un no-saber; la comunicación cotidiana revela que con el formato de pregunta se hacen muchas cosas además de interrogar (expresar sorpresa, suavizar órdenes, pedir consentimiento, inducir respuestas, evaluar, etc.).

El instrumento es, entonces, más afirmativo que interrogativo. Y como son instituciones serias las que lo respaldan, se puede pensar que ese listado (de afirmaciones con signos de interrogación), que describe aquello de lo que dependerían los desempeños de los

2 El formulario de factores asociables aparece inmediatamente después de la prueba de competencias en lenguaje, en el mismo cuadernillo. estudiantes, proviene de fuentes de alta fidelidad. De ahí que cuando alguien interroga por esos instrumentos, inmediatamente se argumenta que han sido validados ya por algún organismo internacional, o que las investigaciones Y y Z han demostrado la asociabilidad de tales factores.

Que se trata de una descripción y no de una carencia de saber (que justificaría el apelativo de investigación para el proceso de evaluación masiva), lo prueba el hecho de la delimitación que produce el cuerpo de "preguntas": ¿por qué esas y no otras? Cuando se trata de delimitar qué investigar, el mismo Ministerio de Educación Nacional (MEN) señala factores sobre los que sería posible hacer otras preguntas (y otros tipos de preguntas), pero que los descarta sin haber investigado, solamente porque ya sabe qué va a encontrar. En nuestra investigación (Bustamante-Díaz, 2000) mostramos cómo uno de los principales argumentos para caer siempre en instrumentos de factores asociables muy parecidos es el factor técnico: es más fácil procesar preguntas cerradas que preguntas abiertas, independientemente de que haya aspectos cuya percepción requiera preguntas abiertas.

\section{La alusión a lo "personal"}

En primera instancia, se interpela al encuestado: "Para terminar, en esta encuesta vas a encontrar preguntas sobre tu vida escolar y sobre tu familia". En ese sentido, vienen las preguntas "personales":

- ¿Cuántos años cumplidos tienes? [número]

- ¿De qué sexo eres? [se dan dos opciones]

- ¿En dónde naciste?

Así, se toca aquello que supuestamente constituye al encuestado: su identidad. Desde la sencilla perspectiva del llamado a un trabajo colectivo que está materializada en la idea de PEI, uno puede preguntarse si el estudiante debería o no (si es razonable o no) ser una unidad de análisis. Pero, sobre esto el mismo documento del DNP que se comenta ha dicho otra cosa: cuando plantea los objetivos del proceso evaluativo, no se refiere al individuo, sino a la institución: información sobre desempeño de sus alumnos (promedios) para orientar sus planes de mejoramiento, atención a las instituciones más necesitadas... No es contrario a cierto propósito de la norma educativa establecer que la unidad mínima de análisis sea la institución; el individuo no podría serlo, pues la educación concibe y busca un individuo, sí, pero definido en el marco de un colectivo; desagregarlo 
de ahí, ¿no sería desnaturalizarlo, buscar en un sentido opuesto al interés "comunitario"? Cuando se crea "artificialmente" una unidad de análisis y se recaba información, como las cifras se dejan manipular, algo ocurre (por ejemplo, asociaciones estadísticas, existentes o no, positivas o negativas) que viene a darle, a posteriori, existencia "real" a la unidad de análisis creada (Desrosières, 1995).

En cualquier caso, ese llamado a la "individualidad" (tu vida escolar, tu familia) es aparente: el evaluador solo necesita del encuestado una marca que permita operar con las cifras y asignarlas a los lugares respectivos (informes por institución, por ejemplo). No habría inconveniente en asignarle una marca aleatoria, con tal de que se mantenga de manera sistemática. Contra la individualidad apelada, de los instrumentos diligenciados se obtendrá una estandarización de la población, una identificación de muchas personas sobre la base de los rasgos que el sistema de información hace aparecer como comunes (por ejemplo, niveles de competencia), no sobre la base de sus rasgos específicos. Por eso se hacen afirmaciones como: "Los resultados son preocupantes pues en primaria, se estaría superando muy levemente un resultado insuficiente y en secundaria, sería plenamente insuficiente" (DNP, 2000, p. 9). Es el mismo tono de 8 años atrás: "Los alumnos de los grados tercero y quinto de la educación básica primaria no muestran, en su mayoría, logros que reflejen el nivel educativo en el que están ubicados" (MEN, 1992, p. 97).

$\mathrm{Y}$ es con datos como estos que se opera el cruce con los factores asociables. Con datos individuales es imposible hablar de "factores asociados a la calidad".

Es muy posible que quien conteste sienta que se relaciona de alguna manera con la preocupación por las competencias básicas: puede creer que con sus respuestas (lo que incluye sus omisiones) contribuye, o que hace una resistencia - pasiva o activa - y, si no entiende, ya se está preparando mediante estos mecanismos para que mañana entienda de cierta manera. Todo esto se asegura, entre otras, mediante la enunciación: "Te demandamos información privada, lo cual te hace sujeto y te define en cuanto individuo de una clase a la que también pertenecen otras personas que llenan el mismo instrumento". Los instrumentos devuelven, a los que entran en relación con ellos, certeza acerca de su identidad (colaborador, investigador, luchador, resistente, asesor, funcionario, etc.) y acerca de una realidad aparentemente exterior a los instrumentos: la educación. En verdad, una y otra no son más que el efecto de esas interacciones, con ayuda de esos instrumentos y gracias a las expectativas que se les enlazan. Lo interesante es que esto desborda toda intención. Si se preguntara desde tal perspectiva, cambiaría radicalmente todo el cuestionario.

\section{"Es mejor permanecer"}

Se pregunta reiteradamente por la permanencia en las instituciones educativas:

- Desde que empezaste primaria, ¿en cuántos colegios has estudiado?

- ¿El año pasado estudiabas en este colegio?

- ¿Cuál o cuáles cursos has repetido?

- Este mes, ¿cuántos días has faltado al colegio?

- ¿Cuál fue la principal razón por la que no viniste esos días?

Tanta insistencia tal vez se deba a que, de antemano, se quiere que los resultados de la investigación coincidan con los criterios de eficiencia y eficacia que ya forman parte de la política educativa. A esas nociones están unidas las ideas de retener a toda costa a los estudiantes, de eliminar la deserción, la repitencia y el ausentismo. Está en todos los documentos oficiales y, por supuesto, en el Plan Sectorial del D.C.. Pero no es esta una iniciativa nacional: las entidades internacionales que financian la educación de países como el nuestro, exigen, impulsan y financian la investigación educativa que incluya esos aspectos, pues se pretende reducir los gastos del Estado, lo que implica atacar fenómenos como la deserción y la repitencia - visibles y problemáticas desde esa mirada-, que reduplican los gastos. Todo ello se justifica si hay un nexo entre desempeño y permanencia-promoción, con lo que el formulario estaría afirmando: "la permanencia es un factor asociado al logro educativo" y, por lo tanto, condenando la no permanencia (sin importar aquello que la cause, sin ocuparse de aquello que la cause).

Con las preguntas, entonces, queda dicho que permanecer en la institución influye en el rendimiento. La asociación estadística vendría a ser una "prueba":

En todos los grados evaluados, a mayor edad del alumno, menor logro [...]. Sistemáticamente los estudios de factores asociados han reportado [...] las repercusiones negativas del ausentismo escolar, la repetición, la deserción y una mayor movilidad escolar, en el logro escolar. (DNP, 2000, p. 11)

Pero sabemos que esta "prueba" en realidad consiste en una correlación numérica que pide una teoría que explique lo que sucede allá en la educación; no es un argumento educativo. De ahí que aparezca 
todo un "imaginario explicativo", extraeconómico, pero que resulta necio y fácilmente objetable: no es cierto que permanecer en la escuela signifique más "juicio", menos "pérdida" de años, más "arrepentimiento", más "adaptación”, más aprendizaje... y que deambular signifique ser indisciplinado, perder años, aprender menos, ser "altanero", desadaptado.

Lo interesante es que las acciones terminan haciendo existir esas nociones pueriles: se prohíben o se estorban los reingresos a la institución, por las causales de "mala conducta", "ausentismo" o "bajo rendimiento", con lo que se obliga a cambiar de institución, para luego afirmar que ese movimiento es indicador de conductas "indeseables". Puede ser adagio popular, pero no es necesariamente cierto que "la constancia vence lo que la dicha no alcanza". El momento de la obtención de los datos está puesto cuando ya el efecto perverso de la creencia ha promovido a la existencia la situación que ha de ser "detectada".

Así, la fidelidad a la institución, el privilegio al proyecto de todos, la exaltación de la permanencia, se consideran "valores" y se promueven. Y entonces se pregunta en consecuencia. No se entiende la imposibilidad de la fidelidad a la institución, en tanto ella tiende a la permanencia (de ahí la idea de perfil y el "gentilicio" institucional: "javeriano", "lasallista", etc.), mientras que el propio proyecto puede ser cambiante e indefinido; puede verse impulsado por un proyecto colectivo, pero también puede verse obstaculizado. El "todos" de las instituciones educativas puede ser una disminución intelectual (cf. Freud, 1990), además de que, en los momentos críticos, suele desdibujarse para dar lugar a intereses hasta entonces ocultos, siendo que mientras tanto se hacía aparecer y se valoraba el sentimiento de la culpa (para aquel que no se inscribe), basado en la consideración de que, como las cosas se hacen "por tu propio bien", si no funcionas eres "malo", "desagradecido", "sinvergüenza", etc. Y como no se concibe que el cambio sea una condición del conocimiento y de los valores prácticos, entonces se promueven "valores" a-históricos.

Si se pensara en la utilidad del cambio y de quien cambia, tanto para sí mismo como para los demás, en la necesidad de valorar esa especificidad en los espacios escolares, las preguntas para establecer factores asociados serían muy distintas. Visto desde esta perspectiva, podría decirse, por ejemplo, que el muchacho no se va de la institución, sino que hay una expulsión simbólica; y, entonces, en lugar de contabilizar el número de colegios por los que ha pasado el niño, podría interrogarse por las prácticas de expulsión simbólica presente en esas instituciones. El formula- rio, entonces, legitima concepciones que no quieren ser tocadas: de educación como favor, de ética como sacrificio de lo individual en favor de lo colectivo.

La búsqueda de factores asociados a la calidad piensa la institución educativa como un bien; mientras esto no se interrogue, se seguirán encontrando ciertas cosas. Pero, ¿acaso no podría pensarse, más bien, en la institución como una tensión entre el proyecto colectivo y el proyecto personal? (Saldarriaga, 2000). Dirección hacia la cual no pueden apuntar los actuales instrumentos pues, frente a lo "personal", ya vimos qué se busca; y frente a lo institucional se intenta imponer la idea de gestión. Pero si pensara que, pese a tener una inscripción en la institución, el sujeto tiene permanentemente otras inscripciones en lo social, otros serían los factores a asociar. Igual análisis para la institución: ella juzga hacia adentro las fidelidades y las infidelidades, pero, ¿acaso no está desdibujada hacia múltiples puntos externos?: lo administrativo, lo social, lo académico, lo disciplinar... todo señala su "definición" en múltiples sentidos que trascienden la "visión", la "misión" y cuantas palabras alimentan la ilusión de que la danza de la lluvia produce la lluvia 3 .

\section{E1 "aprestamiento"}

En el formulario se pregunta: "Antes de entrar a primaria, hiciste [jardín, kínder, grado cero]". Esta pregunta es típica: no tiene pierde, siempre saldrá asociada: "Sistemáticamente los estudios de factores asociados han reportado los beneficios que ofrece el preescolar para los logros académicos posteriores" (DNP, 2000, p. 11). La asociación es sistemática... iy sin embargo se sigue haciendo la pregunta una y otra vez en las evaluaciones masivas! Y la asociación aparece no porque haya asociación, sino porque la escuela se ha organizado para ello y se pregunta en tal dirección. Sabemos que en Colombia -incluso a escala mundial- el aprendizaje de la lectura y de la lengua escrita es un problema. Pero, ¿problema para quién? La investigación del grupo encabezado por Ferreiro y Teberosky (1979) ha demostrado que, contra todo pronóstico, el problema no es de los niños, sino de una enseñanza que no entiende la lectura y la escritura como dos objetos de conocimiento, sino como "necesidades" que el niño no sabe que tiene. En lugar de eso, según las mencionadas investigadoras, el niño construye conocimiento mediante: a) sus

3 Refiriéndose a lo bueno y lo malo de la planeación estratégica, Dartmouth Brian Quinn (citado por Harari, 1996) dice que la danza de la lluvia "no ejerce ningún efecto sobre el clima de los días subsiguientes, pero quienes participan en el ritual creen que sí". 
acciones sobre los objetos del mundo; b) la confrontación de los resultados de sus acciones, con sus propios conceptos y explicaciones; c) la confrontación de los resultados de sus acciones, con los conceptos y explicaciones de los otros; d) la confrontación de los resultados de las acciones de los otros, con sus propios conceptos y explicaciones; y e) la confrontación de sus propios conceptos y explicaciones con los conceptos y explicaciones de los otros.

Su experiencia durante las últimas tres décadas del siglo pasado fue contundente: en lugar de señalar un déficit del niño, encuentran que cuando un maestro es investigador de su propia práctica de enseñanza de la lectura y de la lengua escrita, los niños aprenden y el maestro comprende. Pero, en lugar de continuar promoviendo esta idea - para lo cual el MEN publicó el texto Enseñanza de la lengua escrita y de la lectura, del grado cero al grado tercero-, ahora volvemos a achacarle el problema del fracaso escolar y de los bajos niveles del rendimiento estudiantil (de las competencias, se dice ahora) a lo ocurrido en los primeros grados; así, en lugar de interrogar la enseñanza en esos niveles, se crean otros niveles previos: la Constitución Política de Colombia habla de ¡tres niveles preescolares! Con eso, cuando el niño llegue a primero ya sabe leer y escribir y se traslada el problema a los grados cero o preescolar, donde la enseñanza no ha sido cuestionada, donde la pretensión del sistema permanece.

Así, el instrumento de factores asociables pregunta a los niños si hicieron jardín, kínder o grado cero. Los que respondan afirmativamente, por supuesto, saldrán "asociados" a mejores logros, con lo que el sistema dirá: "queda demostrado que está muy bien la política de grado cero, que está muy bien enseñar a leer y a escribir como condición para llegar a primero". De donde el o los años previos de preescolar juegan como experiencia previa frente a pruebas que presuponen saber leer y escribir. Desde otra perspectiva, interrogando por la práctica de la enseñanza de la lectura y de la lengua escrita, así comenzáramos en primero, los resultados serían distintos... Y otros factores saldrían "asociados".

\section{"Es mejor no emigrar"}

En el cuestionario se pregunta: “ ¿Hiciste primero de primaria en Bogotá?". Como preguntas se hacen pensando en algo, dijimos ya que se trataba de un discurso que afirma: "las competencias dependen de.... De modo que, según esta pregunta, las competencias dependen de si uno ha nacido o no en Bogotá: "Los nacidos en Bogotá tienen mayor logro que sus compañeros inmigrantes. Sin embargo, cuando esa inmigración no es reciente han tenido una mayor oportunidad de adaptación, superando a quienes inmigraron posteriormente" (DNP, 2000, p. 11).

En otras palabras: "No es bueno emigrar a Bogotá, quédate en tu pueblo"; o, en su defecto: "Si no puede evitar venirse para Bogotá, hágalo con suficiente antelación a los exámenes de competencias básicas". Lo que no se dice, y no se busca como gobierno superar, son las razones por las cuales la gente se ve obligada a emigrar de su región hacia Bogotá. De nuevo, se trata de una corroboración de algo dicho atrás: el sistema se declara incapaz de transformar las causas sociales de la diferencia educativa y procede como si las causas fueran otras. Pero, ¿esto conduce a cambiar cosas importantes? ¿Qué sacamos con mejorar una cuarta parte del rendimiento (p. 39) de un niño inmigrante, mejorando el "efecto colegio", si su familia viene huyendo del campo por persecución política, asunto que puede hacer, por ejemplo, que el "efecto colegio" se pierda porque ese niño tiene que seguir huyendo o porque tiene que dedicarse a trabajar o porque lo asesinan? ¿No sería mejor eliminar ese efecto "desplazados" que incrementar el "efecto colegio"?

Atención con la terminología: "inmigrante"; y, sin embargo, se trata de un colombiano. Hasta donde sabemos, esa condición no lo obliga a estar en cierta región; pero, si en realidad es algo importante, y como sabemos que algunos de los asesores de estas medidas de evaluación masiva y de definición de políticas educativas no son bogotanos, ¿se aplica a ellos la idea de quedarse en su pueblo?, ¿será que no son tan lúcidos como los bogotanos?, ¿o será que ya se "adaptaron"?

Supongamos que es cierto que un niño inmigrante tiene menos rendimiento; pero explicar esto con base en la noción de "adaptación" deja mucho por decir. Es como si no hubiera existido todo el constructivismo durante cinco décadas del siglo pasado, con todas sus incidencias en la educación, promovidas por quienes hacen la política educativa. Por ejemplo, si una institución necesita "adaptación", ¿qué es lo que tiene de proyecto?, ¿cómo asume la diversidad? Porque parecería que estar desadaptado es no poderse acoplar a los distintos PEI que, en principio, se declaran respetuosos de la diferencia. Con una Ley General de Educación "progresista", seguimos como a comienzos del siglo pasado: los niños tienen que adaptarse. El conductismo sigue siendo la filosofía y la psicología de quienes diseñan $-o$ contribuyen a diseñar- la política educativa. 
Qué buena prueba para nuestra institución educativa: no es capaz de asumir la diferencia, causa, entre otras, de la necesidad de emigración. Es decir, las causas que llevan a un niño a emigrar pueden ser -al menos parcialmente- las mismas que lo hacen "inadaptado" a la escuela. Lo que el sistema quería botar por una puerta, se le entró por la otra. Lo que pasa es que, en la nueva situación, el problema es achacable a asuntos de poca monta, e incluso, al niño; de ahí ideas como la de "adaptación", o la de "problemas de aprendizaje”.

\section{"La calidad depende de la distancia, pero depende..."}

En el cuestionario se pregunta:

- ¿Cuánto tiempo gastas para venir al colegio?

- ¿En qué vienes al colegio todos los días?

Al hacer estas preguntas, se dice que hay correlación entre desempeño (competencias básicas) y el factor tiempo-transporte: se espera que el desempeño vaya mejorando a medida que no se emplee demasiado tiempo en llegar al colegio, pero en función del tipo de transporte (de ahí la segunda pregunta), pues lo que se demore el desplazamiento introduce un tiempo que puede ser bastante considerable: según las opciones ofrecidas por la pregunta - hasta media hora, hasta una hora y más de una hora-puede implicar más del $20 \%$ del tiempo de estudio, con sus efectos concomitantes: cansancio al llegar a la institución, desánimo para ir, para regresar, para hacer tareas al llegar a la casa, etc., todas ellas contraproducentes para el desempeño. Sentido común, todos de acuerdo. Por eso está en el instrumento. Y queda dicho, independientemente de que la correlación se mencione (DNP, 1999) o que, después, desaparezca (DNP, 2000).

Los extremos de la correlación serían: el logro es menor cuando se vive lejos y el recorrido implica desgaste mental y físico (las opciones de la pregunta al respecto son: a pie, en bicicleta, otro). Y el desempeño mejora cuando se llega en un tiempo prudencial y se va de manera que no hay tanto desgaste mental ni físico (las opciones de la pregunta al respecto son: carro particular y el transporte del colegio privado).

Este juego, en apariencia necio y un tanto cínico, tiene un trasfondo evidente: ¡se trata de diferencias socioeconómicas! Por eso cuenta menos el llegar rápido cuando la casa está frente a la institución educativa, pero esta es una escuelita pública. Se corrobora lo que sabemos: a los que tienen cómo, les va mejor, y a los que deben tomar buses públicos, educación pública, atención pública, caminar o usar vehículos de auto-tracción (o sea, los que no tienen cómo), no les va tan bien. Pero, ¿está dispuesto el Estado a garantizar que los niños tengan una institución escolar buena y cerca? No, porque estamos en una democracia y la gente puede inscribir su niño donde quiera. Pero al cabo del tiempo de haber hecho esa elección, resulta que:

- Quienes tienen buenos desempeños en las pruebas de competencias no están en la educación pública y, curiosamente, su colegio está a las afueras de la ciudad.

- Y quienes tienen desempeños bajos en las pruebas de competencias, se corresponden con aquellos que hacen filas frente a la institución educativa pública cercana, desde la noche anterior a la apertura de inscripciones (casos sin dispositivo "tome un número"); y que, al no clasificar, deben buscar cupo cada vez más lejos.

Dado el caso de que a un colegio oficial de bajo nivel económico le vaya bien, se tratará - como aclara el DNP (2000, p. 24) - de una excepción: el alto rendimiento de algunos colegios oficiales de bajo nivel socioeconómico "se observa en casos individuales y no como característica del sector".

El gobierno del D.C. no quiere -él dirá que no puede- ocuparse de la pobreza y de sus causas. Sabe que ella produce un elemento negativo para el rendimiento, pero solo se propone una curiosa equidad: que los puntajes se acerquen, pero no las posibilidades sociales. Cuando la cuarta parte del rendimiento que el documento le atribuye a la institución pase efectivamente a verse reflejada en los desempeños, los puntajes serán "menos inequitativos", pero de todas maneras serán distintos, porque esas otras causas de la inequidad, las principales (¿las tres cuartas partes restantes?), permanecen.

Si el tiempo de llegada a la institución educativa y la manera de llegar influyen sobre el rendimiento,

- ¿Se debe agilizar el tiempo?

- ¿Se deben entregar vehículos?

- ¿Se deben prohibir los vehículos particulares?

- ¿Se deben cambiar de lugar las escuelas?

- ¿Se debe dejar de hacer esa pregunta en los próximos instrumentos?

- ¿Se debe preguntar por el modelo de escuela en la que eso se solidifica? 
- ¿Se debe modificar la sociedad en la que las diferencias sociales implican diferencias de tiempo y modalidad de llegada a la escuela?

Quedan muchas preguntas para elaborar instrumentos de factores asociables, pues no es descabellado pensar que la organización de la escuela hace que factores como el que se comenta se vuelvan contraproducentes (o sea, que no lo son por sí mismos). Si el dispositivo educativo reinterpreta la heterogeneidad de factores que inciden sobre el rendimiento, ¿qué preguntar en los instrumentos de factores asociables? La idea de unos factores "asociados" es bastante determinista: si A P B. Pero también la política educativa cree en las iniciativas, pues, por ejemplo, quiere acercar los puntajes entre los sectores socioeconómicos vía el "efecto colegio". En otras palabras, los factores "asociados" no lo son tanto. $\mathrm{O}$, en otras palabras, se asocian a condición de que se den ciertas circunstancias, y por eso la SED quiere crear otras condiciones, incluso otros factores que se asocien positivamente. Entonces,

- Es necesario encontrar las jerarquías entre factores. Actualmente, cuando no se superponen los análisis, se hacen subordinaciones, pero bajo modelos (como los "modelos jerárquicos" de Díaz, 1999) plagados de presupuestos que no se discuten y que son bastante discutibles; o sea que hay que debatir el modelo bajo el cual se proponen jerarquías.

- Es necesario producir los factores. Actualmente, cuando no se contradicen entre sí las políticas, la SED y el MEN lo hacen, pero no lo reconocen, pues suelen hablar desde la "objetividad" de la "detección", tal vez debido a que reconocerse como factor es volverse susceptible de evaluación.

Lo que hace evidente que en este tema lo principal son los intereses en juego: ¿qué se quiere hacer de la educación? Recordemos que muchos de los "factores" hoy existen porque el mismo Estado los puso. La pregunta no solo es para los que diseñan o asesoran la política educativa, sino también para los maestros, los directivos, los padres, los estudiantes. No es evidente, entonces, el asunto. Se trata, en cada caso, de una decisión política, ética, social, y, por lo tanto, opuesta a otras alternativas, no es única, lógica, natural.

\section{"Tarea: hacer tareas"}

A propósito de las tareas, el instrumento interroga así:

- ¿Te ponen tareas de matemática para hacer en la casa?
- En el colegio, ¿te revisan las tareas de matemática?

- ¿Te ponen tareas de lenguaje para hacer en la casa?

- En el colegio, ¿te revisan las tareas de lenguaje?

- ¿Cuántas horas dedicas a hacer tareas en tu casa?

- En tu casa, ¿te ayudan con las tareas?

- En tu casa, ¿te revisan las tareas?

- Para ayudarte a hacer las tareas, en tu casa tienes [listado de material impreso]

- ¿Tienes un cuaderno de correspondencia o de control de tareas?

- En tu casa, ¿te revisan el cuaderno de control de tareas?

Para cada pregunta, se dan escalas de tiempo, de frecuencia, u opciones dicotómicas. Las preguntas afirmarían: "Las tareas están ligadas positivamente al desarrollo de competencias". Si no, la escuela no pondría tantas, y la SED no preguntaría tanto sobre el tema. Dicho y hecho:

La frecuencia de tareas para la casa y la frecuencia de su revisión por parte del profesor, tienen una correlación positiva con logro en matemáticas y lenguaje en primaria. En secundaria, la correlación es significativa en matemáticas. Y mientras un mayor tiempo de dedicación a estudiar y hacer tareas en casa mejora el desempeño académico, recibir ayuda en casa para hacerlas, mostró una asociación negativa en ambos niveles. Es necesario precisar el alcance de este resultado. (DNP, 2000, p. 12)

Lo curioso es que aquí "tarea" significaría lo mismo en todos los casos. Pero, ¿acaso no hay diferencia de un colegio a otro? Claro que la hay, el documento la reconoce: "El plantel explica una cuarta parte de la variabilidad del logro [...] es amplio el margen del desempeño escolar que depende de la calidad de la oferta educativa de las instituciones" (DNP, 2000). Es decir, para unas cosas, hay un factor aleatorio (así lo define el documento) que corresponde a la institución; si eso es cierto, no es un error pensar que, entre otros, esas diferencias tienen que ver con la manera como la institución asigna las tareas, las funciones que éstas tienen, el papel que ocupan en los propósitos de la institución, etc. En consecuencia, sobre la base de los datos aportados por el mismo documento, no se pueden promediar 
las respuestas sobre tareas, porque ese aspecto es fundamental para definir la especificidad de la institución, que tan importante resulta para buscar factores asociados (es casi que su razón de ser, pues constituye el ámbito en el que la política educativa puede actuar, ya que el resto se lo deja a la historia).

Pero, si observamos con cuidado, la cita del DNP lo sabe, pues termina diciendo: "Es necesario precisar el alcance de este resultado". ¿Se justifica el esfuerzo, humano y fiscal, para encontrar que la cosa hay que precisarla? El DNP no dice que son preguntas necias, entonces él mismo se encarga de precisar la cosa:

\begin{abstract}
De hecho, un examen más detallado utilizando el método prinqual, muestra un hecho interesante. Este método permite transformar las valoraciones iniciales dadas (por el investigador) a las categorías de respuesta en valoraciones óptimas, las cuales, conservando el orden entre las categorías, establece la distancia precisa entre ellas. Los resultados de este ejercicio muestran: 1) valoraciones óptimas iguales para todas las categorías de tiempo de dedicación a estudiar en casa lo que significa que, más que el tiempo de dedicación a estudiar en casa, lo que diferencia es simplemente dedicar tiempo o no a estudiar 2) valoraciones crecientes a las categorías de frecuencia con que recibe ayuda para hacer las tareas. Por lo cual, más que afirmar que ayudar a los hijos en sus tareas es negativo para su rendimiento escolar lo que marca la diferencia, es la frecuencia con que lo hace: quienes respondieron que reciben ayuda siempre o casi siempre son los que tienden a tener menor logro. Como se verá en la II Parte, el problema se debe discutir en el marco de la concepción de las tareas y de la forma de ayuda que recibe el niño. (DNP, 2000)
\end{abstract}

Estamos completamente de acuerdo con el DNP. Pues sus afirmaciones nos permiten corroborar algo en lo que hemos estado insistiendo: que con los mismos datos se puede afirmar lo que uno quiera. Por eso:

- Antes de utilizar el método prinqual, la frecuencia de tareas y de revisión tiene una correlación positiva con logro,

- Después de utilizar el método prinqual, la frecuencia no está asociada, sino dedicar o no tiempo a estudiar.

$¡ O$ sea, todo lo contrario! Ante este hallazgo, creemos que cualquier persona, sin invertir tantos recursos, sabe que dedicar tiempo a estudiar influye positivamente en el logro. Como estudiar es una condición de posibilidad del desempeño, no hay que buscarlo mediante una investigación, pues es una necesidad lógica (así como no hay que buscar las "ideas verdes transparentes que duermen furiosamente" ${ }^{\prime 4}$ para demostrar que no existen, pues la idea constituye una contradicción lógica en sí misma).

De otro lado:

- Antes de utilizar el método prinqual, recibir ayuda en casa para hacer tareas muestra una asociación negativa,

- Después de utilizar el método prinqual, hay una asociación positiva para quienes reciben la ayuda con mayor frecuencia.

¡O sea - de nuevo-, todo lo contrario! Igualmente, ante esto nos parece que no hay que entrar en los gastos e investigaciones en cuestión para decir que la ayuda ayuda. Claro, porque, si no, no sería ayuda. Perogrullo estaría dichoso.

Lo más interesante de todo es que con cualquier resultado, así sea opuesto a otro obtenido con los mismos datos, se puede hacer política educativa:

- Que se continúen poniendo tareas, no importa con qué modelos pedagógicos, ni en el marco de qué propuesta de la institución; lo importante es que no se cuestione el modelo de escuela que reposa en el propósito gubernamental y que autoriza la investigación y la intervención.

- Que los padres no ayuden porque sus niveles de desempeño son inferiores a los de los niños. Sabemos a quién va dirigido esto: a los padres deprimidos económicamente (y por eso hay preguntas sobre el nivel académico de los padres). Y que los que en realidad tienen capacidad de hacerlo, lo hagan.

Compartimos totalmente el fin de la cita anterior: "Se debe discutir en el marco de la concepción de las tareas y de la forma de ayuda que recibe el niño", que es justamente el que desautoriza el estudio en mención. Ya lo dijimos: no se puede promediar algo tan específico: es necesario saber qué quiere decir tarea, y qué quiere decir ayuda, en cada caso. Y, una vez más, el DNP está de acuerdo: se necesita.

1) una nueva estrategia metodológica en el estudio de los factores asociados y 2) la realización de investigaciones específicas en algunos temas sobre los que dichas evaluaciones continúan insinuando explicaciones de carácter hipotético. En otras palabras, es momento de dar el salto a nuevas formas de evaluación y estudio, que llenen los vacíos de información que los resultados generales hacen evidentes. (DNP, 2000, p. 62)

4 Se trata de un ejemplo que Chomsky ha hecho famoso en la lingüística. 
Es lo que hemos dicho desde el comienzo de las evaluaciones masivas. Ojalá de ahí en adelante, como promete el DNP, se hubiera hecho realidad ese propósito. Pero, como era de esperarse, estamos condenados a repetir estos procedimientos. Por supuesto que no se trataría solo de cambiar una "estrategia", sino fundamentalmente de interrogar una posición desde la que se habla; por ejemplo, el DNP aclara en primera instancia que "es necesario precisar el alcance de este resultado", pero ha hablado de correlaciones entre cifras como hechos en la realidad educativa (es que no aplica su mismo enunciado de que se trata de hipótesis, no de hechos). Y, en segunda instancia, habla de discutir la concepción de tareas y de ayuda, pero no menciona la discusión de la concepción de escuela en la que se basa la evaluación masiva y la indagación de factores asociables.

\section{¿Factor "asociado" o perspectiva?}

Para ilustrar la importancia de la perspectiva desde la que se interroga por un factor, retomaremos una de las preguntas del punto anterior, la relativa al material impreso:

- Para ayudarte a hacer las tareas, en tu casa tienes:

\begin{tabular}{|l|c|c|}
\hline Enciclopedia & Sí & No \\
\hline Libros de literatura o cuentos & Sí & No \\
\hline Diccionario & Sí & No \\
\hline Otros libros & Sí & No \\
\hline
\end{tabular}

Todos los instrumentos para buscar factores asociados al desempeño se preguntan por el material impreso, pero cuando no lo hacen de manera estereotipada y repetitiva de unos a otros, lo hacen desde perspectivas distintas, incluso opuestas. Pues bien, estas perspectivas son fundamentales: ellas determinan las maneras de preguntar $y$, por consiguiente, los "hallazgos":

Mientras en 1992, durante la evaluación de la calidad del SINECE, se pregunta:

- En tu casa, lees libros distintos de los textos escolares:

\begin{tabular}{l} 
¿Muchas veces? \\
\hline ¿Rara vez? \\
\hline ¿Nunca?
\end{tabular}

En la evaluación de impacto del Plan de Universalización de la Educación Básica Primaria esto se indaga de la siguiente manera:

- ¿Cuántos libros hay en tu casa? (No incluyas las revistas ni los periódicos)

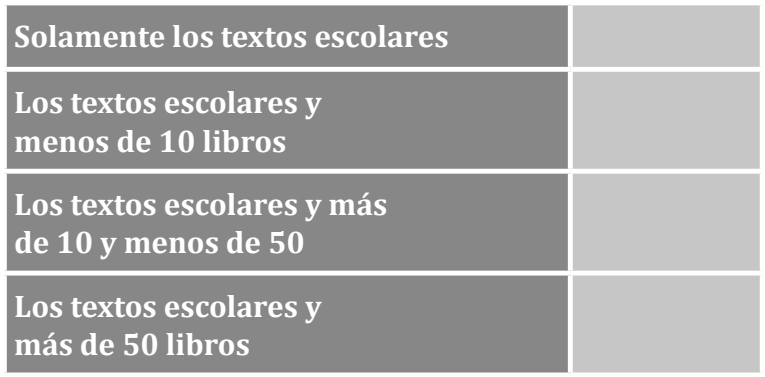

Comparemos solo la literalidad de las tres preguntas; sin ir más allá, parece haber más de una concepción sobre el material impreso (tabla 1).

Tabla 1.

\begin{tabular}{|c|c|c|c|}
\hline & SINECE & Universalización & 1998 \\
\hline Año & 1992 & 1997 & Nonsal \\
\hline $\begin{array}{c}\text { Texto escolar es dife- } \\
\text { rente de libros }\end{array}$ & Sí & Sí \\
\hline $\begin{array}{c}\text { Revistas y periódicos son } \\
\text { materiales impresos }\end{array}$ & $\begin{array}{c}\text { Considera los periódi- } \\
\text { cos en otra pregunta }\end{array}$ & No & $\begin{array}{c}\text { Deja la posibili- } \\
\text { dad de "otros" }\end{array}$ \\
\hline $\begin{array}{c}\text { Unificar material impreso } \\
\text { en la escuela }\end{array}$ & Sí & Sí & No \\
\hline Tenencia de libros & No & Sí & No \\
\hline Uso de libros & No & No & Sí \\
\hline Frecuencia de lectura & Sí & No & No \\
\hline
\end{tabular}


Leyendo horizontalmente, vemos que en ningún caso hay coincidencia, ¿cómo pensar, entonces, que se tiene información confiable para proceder al trazado de la política educativa? Sin embargo, los estudios existentes se usan (o se mencionan) para hacer comparaciones, para tomar decisiones... Por supuesto, no se trataría de que hubiera una lista de factores constante o listas equivalentes, sino de posicionarse frente las concepciones que en cada caso dan lugar a los instrumentos. La perspectiva investigativa, que tanto se pregona, implicaría partir de cierta incertidumbre, enunciar con cierta reticencia, buscar como resultado de que existen preguntas y no sólo la intención de imponer cierta política educativa. En lugar de eso, y a nombre de la investigación, tenemos la idea de imponer un sistema de evaluación, lo que implica partir de certezas, enunciar como dueño de la verdad y tener resultados antes de las búsquedas. Así, el sistema legaliza una política educativa no del todo legítima, mientras la investigación podría soportar una modesta y abierta política educativa. Estas asimetrías se resumen en la tabla 2.

Tabla 2.

\begin{tabular}{|c|c|}
\hline \multicolumn{2}{|c|}{ Perspectiva } \\
\hline Investigativa & $\begin{array}{c}\text { Imposición de cierta } \\
\text { política educativa }\end{array}$ \\
\hline $\begin{array}{c}\text { Concertar un sis- } \\
\text { tema de evaluación }\end{array}$ & $\begin{array}{c}\text { Imponer un sis- } \\
\text { tema de evaluación }\end{array}$ \\
\hline $\begin{array}{c}\text { Partir de cierta } \\
\text { incertidumbre }\end{array}$ & Partir de certezas \\
\hline $\begin{array}{c}\text { Enunciar con cierta } \\
\text { reticencia }\end{array}$ & $\begin{array}{c}\text { Enunciar como estando } \\
\text { en posesión de la verdad }\end{array}$ \\
\hline $\begin{array}{c}\text { Buscar como resultado de } \\
\text { que existen preguntas }\end{array}$ & $\begin{array}{c}\text { Tener resultados antes } \\
\text { de las búsquedas }\end{array}$ \\
\hline $\begin{array}{c}\text { Soportar una modesta y } \\
\text { abierta política educativa }\end{array}$ & $\begin{array}{c}\text { Institucionalizar una } \\
\text { política educativa }\end{array}$ \\
\hline
\end{tabular}

\section{"Procure no ser pobre I"}

A propósito de la composición del hogar, el instrumento pregunta:

- ¿Cuántas personas en total viven en tu casa? [número]

- ¿Cuántas de esas personas son de tu familia? [número]

- Las personas de tu familia, ¿en cuántos cuartos duermen? [número]
- ¿Cuántos hermanos tienes mayores que tú? [número]

- En tu casa vives con: [se dan las opciones]

Estos índices se utilizan para determinar niveles de pobreza y no niveles de riqueza, de manera que no habría niveles de riqueza, no hay que buscarlos. Entonces, es claro que se enuncia desde la riqueza. No son indicadores exclusivos de instrumentos de factores asociables a la educación, pues se utilizan también para establecer índices de necesidades básicas insatisfechas. Queda claro que los indicadores que se están usando en educación provienen de la política social y no de un análisis en el sector educación, ni de una construcción de ese sector; es decir, el modelo político aplana deliberadamente las especificidades (por ejemplo, salud y educación) a favor de una manera de juzgar su eficiencia interna.

El informe del DNP (2000, p. 12), dice que "a medida que aumenta el tamaño del hogar y el hacinamiento, menor es el desempeño escolar". En otras palabras, "si quieres rendir de manera óptima en las pruebas de competencias básicas, procura no hacinarte, no ser pobre". Es otra manera de decir que la variable de mayor correlación positiva con el logro es el nivel socioeconómico del hogar. Pero, ¿acaso se está dispuesto a tomar decisiones sobre este aspecto? Ni el niño es "culpable" del nivel socioeconómico de su hogar, ni puede mejorarlo voluntariamente; pero tampoco la política educativa del D.C. muestra voluntad de modificarlo para favorecer el desempeño. Entonces, ¿qué sentido tiene verificar, una y otra vez, que la gente es pobre?, ¿qué sentido tiene señalar esto como causa principal de las diferencias de desempeño en las evaluaciones masivas?, ¿sobre todo si de lo que se trata es de afectar la porción en la que el colegio incide? Estas posturas le dan cabida a investigaciones tales como: La importancia del agua en la navegación acuática.

Si la pobreza es causa principal de las diferencias de desempeño, la política que se desprende es atacar la pobreza, no describirla y pasar a tratar de incidir en otros aspectos para que las cifras no sean tan escandalosas. Y, si como dicen los gobiernos, ya no hay opción de atacar ese factor, entonces no hay que seguirlo preguntando y enrostrando.

Desde la misma lógica (condición socioeconómica) se plantean otras preguntas del instrumento:

- La mayor parte del piso de tu casa (o apartamento) es de: [se dan las opciones]

- La mayor parte de las paredes de tu casa son de [se dan las opciones] 
- ¿Con qué cocinan en tu casa? [se dan las opciones]

- En tu casa hay [se dan las opciones de servicios públicos]

Entonces, una estrategia oficial dirigida a "compensar las diferencias iniciales" (DNP, 1998, p. 108), toda vez que se ha declarado la imposibilidad de transformar las estructuras generadoras de pobreza (quedando solamente la opción de disminuir la desigualdad dando acceso a "todos los individuos a una educación de calidad" (p. 98), haría recomendaciones como la siguiente: "Si quieres mostrar buenos desempeños en las pruebas de competencias básicas, no vivas en una casa con piso de tierra, con paredes de bahareque, con estufa de cocinol, sin servicios...." Claro, pues no se trataría de la estructura de la sociedad, sino posiblemente de una diferencia de calidad en la oferta educativa, combinada con una diferencia de aprovechamiento de esa oferta educativa.

\section{La escuela, ¿ya estaba en la casa?}

1. Las preguntas sobre alfabetización y nivel educativo de las personas que componen el hogar parecen enunciar la idea de que "la calidad de la educación depende de la medida en que la escuela ya esté presente desde la socialización primaria [la que brinda el hogar]". De por sí, la búsqueda en este sentido tiene que dar correlación positiva. El caso que se comenta no es una excepción: "Los alumnos de hogares con mayor educación de sus padres o acudientes tienden a tener mayor logro" (DNP, 2000, p. 12). De nuevo, como se trata de una condición de posibilidad, no es necesario buscarlo para saberlo (y, sobre todo, reafirmarlo una y otra vez).

Desde mediados del siglo xx, Basil Bernstein dedujo - sin hacer evaluaciones masivas- que el código de la escuela está más próximo a la socialización primaria que brindan ciertas clases sociales que, entre otras cosas, han pasado por la escuela. Cuando el hogar está marcado por la escuela, indefectiblemente hace un ambiente favorable para ella en el niño aún no escolarizado; esto, obviamente, favorece sus desempeños. Otras clases sociales, que no están tan marcadas por la escuela, en su socialización primaria no predisponen favorablemente a sus hijos para cuando ingresen a ella, lo cual desfavorece sus desempeños:

Las pedagogías invisibles dan lugar a procedimientos de control basados en pautas de comunicación multidireccional de clase, necesarias para apoyar y promover su concepto y práctica del orden social. Y es probable que la construcción de estas competencias comunicativas se base en la clase social. Cuando la familia no pone a disposición del niño estas competencias, es menos probable que éste se autorregule en la escuela, de acuerdo con los requisitos de su práctica pedagógica invisible, y es fácil que interprete de forma errónea tanto la práctica como su contexto pedagógico. (Bernstein, 1993, p. 93)

Es redundante buscar evidencias estadísticas para mostrar, por ejemplo, la "importancia de la masticación en la buena digestión".

2. Cuando se pregunta si "Alguien de tu casa asiste a las reuniones de padres de familia", se afirma que "la calidad de la educación depende del apoyo y la preocupación de los padres”. Este enunciado resulta ser un estereotipo acogido por el instrumento y promovido por él. Acoge el estereotipo en cuanto da fe de la idea de que el apoyo de la familia es favorable: "ustedes no tienen buenos desempeños porque tienen padres que no son responsables con ustedes"; pues bien, ya habíamos visto que el mismo documento del DNP dice que no (que cierta "ayuda" perjudica). Pero, según venimos de ver en Bernstein, el papel de la familia ha de ser tomado en términos estructurales y no de "voluntades".

$\mathrm{Y}$ el instrumento promueve el estereotipo en cuanto también sirve para posicionar la idea - propia del modelo político en boga- de que es beneficioso transferir cada vez más las responsabilidades de la educación a la familia: "la calidad educativa tiene correlación positiva con la delegación, hacia la familia, de las responsabilidades estatales frente a la educación".

\section{"Procure no ser pobre II"}

Ante las respuestas a la pregunta por las actividades a las que el estudiante dedica más tiempo cuando termina actividades en el colegio, el informe analiza con profundidad la relativa al trabajo infantil: "El trabajo infantil de manera reiterada y como es de esperarse, muestra efectos negativos en el rendimiento escolar". Pero, ¿por qué "era de esperarse"? Para nosotros, por las razones que venimos esgrimiendo: porque aquellos niños que deben trabajar son aquellos que lo necesitan, es decir, los de nivel socioeconómico bajo. De manera que el trabajo infantil no está correlacionado de manera negativa con el rendimiento, como dice el DNP: en realidad, como el mismo DNP lo explica, el rendimiento está asociado a la condición 
socioeconómica, así como la necesidad de que los niños trabajen. Entonces, sí era de esperarse.

Los niños trabajan cuando en sus hogares no ven satisfechas las necesidades: trabaja el $40 \%$ de las niñas del quintil más pobre y el $23 \%$ de las niñas del quintil más rico (DNP, 2000, p. 13); y, pese a la evidencia de estas cifras, habría que establecer el significado de "trabajar" en cada caso. La opción que se ofrece en el instrumento, "Ayudar en las labores del hogar", significa cosas muy distintas cuando comparamos hogares de estratos distintos: llegar a trabajar en la tienda no es lo mismo que llegar a ordenar que se le atienda.

Que trabajen los niños que lo necesitan, implica que los que no lo necesitan pueden realizar labores más ligadas al mejoramiento de sus desempeños: "Actividades como ver televisión, navegar por Internet o jugar videojuegos se asocian positivamente con logro" (DNP, 2000, p. 13). De otro lado, esto revelaría la existencia de una escuela que no es capaz de incorporar las implicaciones del trabajo en el desempeño de los niños. Una prueba más de que el código de la escuela es más próximo al de la familia que no necesita el trabajo de los niños para satisfacer sus necesidades.

\section{Algunas conclusiones}

- Puede que los instrumentos de "factores asociables" muy probablemente estén midiendo - contra el propósito de las aplicaciones-, la capacidad de los encuestados para inferir la pragmática de las pruebas.

- La forma interrogativa de los enunciados no materializa el tipo de comunicación que establece el instrumento. En realidad, se trata de una serie de afirmaciones sobre la llamada "calidad de la educación" que solo se justifican reenviando a otros estudios y por "factores técnicos" que solo entienden los técnicos. Pero, curiosamente, las preguntas tienen que ver con las medidas de política educativa que se pretenden implementar.

- Se toca la "identidad" del encuestado en la medida en que se convierte al estudiante en "unidad de análisis". Sin embargo, de un lado, el sujeto importa muy poco, pues es solamente un guarismo que debe permanecer constante para poder hacer los "cruces estadísticos" entre desempeño y factores asociables; no en vano, después se hablará de tendencias generales y los informes nunca llegarán a ser individuales. Y, de otro lado, esto contradice la misma política educativa que ordena construir proyectos educativos institucionales y que debe responder, también de manera colectiva, a las implicaciones de la evaluación.

- Buscar la permanencia en la institución educativa como "factor asociado" a la calidad no es más que la manera de hacer un ahorro fiscal, de un lado, sin tener que interrogar - e intervenir - las causas sociales de la deserción, la repitencia y el ausentismo (que muchas veces son, en realidad, expulsiones simbólicas); y, de otro lado, sin dar lugar a la productividad de la movilidad.

- El aprendizaje de las primeras letras y de los rudimentos del cálculo es fundamental para la postura del estudiante frente al conocimiento, y da cuenta de la postura de la institución y de los maestros frente a la enseñanza. Así, buscar la asociación entre desempeño y aprestamiento (años de preescolar) es una manera de empujar hacia atrás el problema (los niños terminan el primer grado con un bajo nivel de lectura, escritura y cálculo) y de no verse obligado a intervenir este asunto trascendental de la educación.

- Muchos de los factores indagados (ejemplo: cuánto se tarda en llegar un niño a la institución educativa, la manera como se moviliza, el nivel educativo de los padres, las características de la vivienda, el número y el parentesco de las personas con quien vive, trabajo infantil...) en realidad están subsumidos en un gran factor: nivel socioeconómico. Todas las preguntas al respecto son redundantes y el efecto es el mismo: la desigualdad socioeconómica produce desigualdad de desempeños escolares. Ahora bien, desde el sector educativo se pretende afectar solamente el porcentaje "propiamente educativo" de las diferencias (que llaman "efecto colegio"), dando por hecho que es imposible tocar las diferencias que explican las restantes tres cuartas partes del desempeño.

- Pese a las diferencias inherentes a las tareas que pueden asignarse a los estudiantes, pese a las diferencias que estas puedan tener de institución a institución, el análisis estadístico promedia todo esto para afirmar la evidencia de que es bueno que los estudiantes se ejerciten en los asuntos propios de los aprendizajes que están teniendo. Sin embargo, parece que este pleonasmo tiene lugar para exhibir un método (prinqual) con el que se puede decir todo lo contrario de los métodos que se aplican 
usualmente para leer las asociaciones. Sorpresivamente, no se entiende con esto la mutua eliminación de los métodos en mención, sino su uso a conveniencia, al punto de llegar a recomendar que padres con bajos niveles académicos no deben ayudar a sus hijos.

- Los estudios estadísticos encuentran correlaciones numéricas, con el fin de detectar puntos a investigar (disminución de incertidumbre) y, ahora sí, establecer las causas. Sin embargo, el gobierno usa las estadísticas arrojadas por estos ejercicios para hablar de causalidad.

- Decidir qué se investiga es un acto de producción de realidad educativa, no de detección de "cosas que están ahí". El gobierno procede como si recién llegara a la educación, como si no hubiera puesto lo que ahora viene a "detectar" con "instrumentos objetivos".

- Los instrumentos de factores asociables se han establecido mediante listados que provienen de manera desigual y arbitraria de otras indagaciones o de conceptualizaciones poco explicitadas.

- No hay novedad alguna en los hallazgos de estos estudios (se repiten aplicación tras aplicación las mismas evidencias de las que incluso se podría partir) y, no obstante, se siguen realizando, sin dar lugar a otro tipo de estudios, aun retrocediendo frente a sus propias aplicaciones (ejemplo: cambia drásticamente el número de preguntas por asuntos económicos; las preguntas de un instrumento a otro sobre el mismo tema - analizamos el tema del material impreso- pueden materializar posturas opuestas y, sin embargo, se los compara como si hubieran indagado lo mismo).

\section{Referencias}

ÁviLA, R. (1999). «Factores asociados al logro educativo». En: Alegría de enseñar $\mathrm{N}^{\circ} 38$. Cali: FES.

BENEDITo, G. (1975). «El método de los tests e inventarios». En: Psicología: ideología y ciencia. México: Siglo XXI.

BERNSTEIN, B. (1993). La estructura del discurso pedagógico. Madrid: Morata.

Bustamante, G. y Díaz, L. G. (2000). «Análisis de algunos factores asociables al desempeño en el área de lenguaje: el D.C. frente al resultado nacional». Informe de investigación (1999-2000). Bogotá: IDEP, 2000. [En 2003 fue publicada por la Universidad Nacional de Colombia, con el nombre de Factores asociables al desempeño de los estudiantes).

CABrera, B. (1999). «Evaluación del sistema educativo: ¿para qué?». En: Archipiélago № 38. Barcelona: Archipiélago.

Desrosières, A. (1995). «¿Cómo fabricar cosas que se sostienen entre sí? Las ciencias sociales, la estadística y el Estado». En: Archipiélago $\mathrm{N}^{\circ} 20$. Barcelona: Archipiélago.

DíAZ, L. G. (1999). Estadística multivariada: análisis y métodos. Bogotá: Universidad Nacional.

DNP. (1999). Evaluación de la calidad de la educación primaria en Santafé de Bogotá 1998 - Factores asociados al logro. Informe. No publicado.

DNP. (2000). Evaluación de la calidad de la educación primaria y secundaria en Santafé de Bogotá. Factores asociados al logro académico. Informe. No publicado.

Ferreiro, E. y Teberosky, A. (1979). Los sistemas de escritura en el desarrollo del niño. México: Siglo XXI.

Freud, S. (1990). «Psicología de las masas y análisis del yo». En: Obras Completas, Vol. 18. Buenos Aires: Amorrortu.

HARARI, O. (1996). «Lo bueno y lo malo de la planeación estratégica». En: Avianca en revista. Bogotá.

MEN. (1992). Saber. Sistema nacional de evaluación de la calidad de la educación. Primeros resultados: matemáticas y lenguaje en la básica primaria. Bogotá: MEN.

MEN. (1997). Factores asociados al logro cognitivo de los estudiantes. Grados 3ㅇ, 5. 1993-1995. Bogotá: MEN.

Oliverio, A. (1998). «El espectáculo de la ciencia». En: Revista Newton.

SALDARRIAGA, O. (2000). «Matrices éticas y tecnologías de formación de la subjetividad en la pedagogía colombiana, siglos XIX y XX». En: Pretextos pedagógicos, $\mathrm{N}^{\circ}$ 9. Bogotá: Socolpe.

SimiAND, F. (1994). «El estadístico debe saber lo que hace». En: Bourdieu et al. El oficio de sociólogo. México: Siglo XXI.

Stuввs, M. (1984). Lenguaje y escuela. Madrid: Cincel-Kapelusz.

VeLÁzqueZ, E. (1995). «Descripción de los cuestionarios sobre evaluación de la calidad de la educación, aplicados por el ICFES en 1992». Bogotá: ICFES. No publicado. 